\title{
Construction of coarse-grained order parameters in nonequilibrium systems
}

\author{
David E. Reynolds \\ ICES, The University of Texas at Austin, Austin, Texas 78712, USA \\ and Department of Physics and Institute for Genomic Biology, University of Illinois at Urbana-Champaign, \\ 1110 West Green Street, Urbana, Illinois 61801-3080, USA
}

(Received 25 September 2008; revised manuscript received 6 May 2009; published 11 June 2009)

\begin{abstract}
We develop a renormalization-group (RG) procedure that includes important system-specific features. The key ingredient is to systematize the coarse-graining procedure that generates the RG flow. The coarse-graining technology comes from the control and operator theoretic model reduction. The resulting "generalized" RG is a consistent generalization of the Wilsonian RG. We apply the procedure to a deterministic nonlinear wave equation (NLWE) with probabilistic initial conditions. We derive the form of the projection operator from the dynamics of the NLWE and then use it to generate the RG flow for the distribution of initial conditions. The probability density of the initial conditions is chosen to be a Boltzmann weight that is quartic in the field variables. In our calculation, we find that in contrast to conventional implementations of the RG, naïve power counting breaks down. We also show that the resulting RG equations are different from those derived from the conventional RG.
\end{abstract}

DOI: 10.1103/PhysRevE.79.061107

PACS number(s): 05.40.- a, 05.10.Cc, 64.60.Ht

\section{INTRODUCTION}

A recurring theme in the study of complex and biological systems is that the systems of interest are open. The nature of the disturbances rendering the system open may be stochastic, structured, or both. Additionally, the stochastic nature of chemical networks, especially those with molecular types in low abundance, ensures that any intrinsic description must account for the structure and type of uncertainty. Intuition from closed systems, with the possible exception of glassy systems [1], suggests that detailed questions are often too difficult to answer and that asymptotic questions are more amenable to theoretical treatments. While for many open systems the knowledge of the temporal asymptotics is both interesting and important, for others such knowledge may not carry any content or even exist. However, just because temporal asymptotics may not exist for open systems, this does not imply that there are not robustly discernible features of open systems. It simply means that the appropriate coarsegrained description(s) must be informed by the structure of the external and internal disturbances and uncertainties.

A large class of open systems may be represented by equations of the form

$$
\frac{\partial \varphi(x, t)}{\partial t}=f(\{\varphi\})+u(x, t)
$$

where $\varphi$ and $u$ are vectors or functions in possibly infinitedimensional spaces. Without $u$, Eq. (1) represents a closed system or a model that describes a system with perfect certainty. With $u$, the system is inherently open subject to additive uncertainty. $u$ represents generic driving as well as a possible noise source to which the system is exposed. If $u$ is generated by a continuous stochastic process, this imposes a specific structure on the noise. Similarly, constraining $u$ to belong to a particular function space but remaining otherwise arbitrary also imposes a specific structure on the noise. The system, as described, may be mapped to a field theory via generalized Martin-Siggia-Rose (MSR) or closed-time-path
(CTP) methods [2-5]. In particular, the path-integral representation for the probability for the system to be in state $\phi$ at time $t$ given that the initial state was $\phi_{0}$ at time $t_{0}$ is given by [6]

$$
\begin{aligned}
P\left(\phi, t \mid \phi_{0}, t_{0}\right)= & \int D \varphi \exp \left\{-\frac{1}{2 \Delta x} \int_{t_{0}}^{t} d \tau \partial_{\varphi} f\right\} \\
& \times \delta\left[u-\partial_{\tau} \varphi+f(\varphi)+\phi_{0} \delta\left(\tau-t_{0}\right)\right] .
\end{aligned}
$$

When the initial state is only known probabilistically, then the probability for the system to be in state $\phi$ at time $t$ is given by

$$
P(\phi, t)=\int D \phi_{0} P\left(\phi_{0}, t_{0}\right) P\left(\phi, t \mid \phi_{0}, t_{0}\right) .
$$

We introduce this representation because later we will explicitly utilize it in the context of applying the renormalization group $(\mathrm{RG})$ to the initial conditions. It is important to note that upon combining Eqs. (2) and (3), integrating out $\varphi$ does nothing to dress the distribution $P\left(\phi_{0}, t_{0}\right)$. This simply reflects the fact that the initial conditions are imposed as a constraint. On the other hand, integrating out $\phi_{0}$ would then add stochastic contributions to Eq. (1).

Suppose $u$ is an arbitrary input to the system that is square integrable in time. We consider the states or regions in phase space that are most accessible via driving to be responsible for describing the essential characteristics of the system. This is analogous to the energy landscape picture in statistical mechanics where fluctuations govern which states contribute the most to the statistics of the system. We use this inherently open systems perspective of the importance of states to specify how to coarse grain and, consequently, to generate $\mathrm{RG}$ equations. The key step in generalizing the $\mathrm{RG}$ lies in ascertaining how to coarse grain.

Although it is already known that the RG is not a black box routine, the purpose of this work is to make it more algorithmic. It is easy to be misled into thinking that the RG 
already is algorithmic because its key ingredients are coarse graining and rescaling the system variables [7-10]. However, fully algorithmic implementations of the RG fail for large classes of problems because it is not possible to ignore the physics of a system and expect to obtain meaningful results. Capturing the essential physics requires isolating the appropriate models and the structure of perturbations and uncertainties. Consequently, this process is system specific. Additionally, the scale on which the physics is observed must be specified. For instance, for bosonic theories the longwavelength physics is investigated, while for fermionic system it is the physics near the Fermi surface. These considerations suggest that the primary obstacles to automation are the model identification and coarse-graining processes. In this paper, we present a RG procedure that accounts for these system-specific obstacles and apply it to renormalize the initial conditions of a nonlinear wave equation (NLWE). We specifically focus on a nonlinear wave equation as opposed to a reaction diffusion equation because the extra time derivative increases the number of the Green's functions that can contribute to the dynamics of the system. As we will see, this can have a significant impact on coarse graining. We do not renormalize the dynamical equations because the structure of the dynamical action renders the perturbative loop corrections uninteresting. The general form of how projection operators, generating the RG, act on the dynamical fields is discussed in [11].

The obstacles mentioned above arise from the (mis)identification of observables. We use techniques taken from operator theoretic interpolation theory and control theory to systematically identify observables that respect the system dynamics. The conventional implementation of the renormalization group relies upon discerning equilibrium observables at predetermined scales. Coarse-graining nonequilibrium systems must respect the nature of the dynamics and the structure of the uncertainty. Emerging fields like systems biology are producing many new problems that are begging for such a treatment $[12,13]$. This work provides a step toward the development of a framework that will be applicable to such systems.

While Eq. (1) addresses the effects of perturbations to the system, it does not account for the consideration that it may not be desirable to describe every observable. For instance, the complete characterization of a system over all scales may carry significant computational overhead. Furthermore, due to experimental constraints, it may not be possible to measure all observables either. Consequently, it is beneficial to explicitly include the possibility of multiscale or constrained observation. One of the simplest cases is when the observables are linearly dependent on the $\varphi$ observables. The description of such open systems takes the form

$$
\begin{gathered}
\dot{\varphi}=f(\varphi)+B u, \\
\psi=C \varphi,
\end{gathered}
$$

where $\psi$ reflects that only some subspace or, more generally, subset of phase space is directly measurable. The operators $B$ and $C$, respectively, specify the structure of how noise may enter the system and which states are considered as observ- ables. More generally, the observables may be nonlinear functions or functionals of $\varphi$; however without mapping the problem to a yet higher dimensional description, that complication is beyond the scope of the analysis presented herein. Considering coarser observables $\psi$ influences the relative importance of the internal states $\varphi$ because many of them simply either do not or only marginally contribute to the physical observables $\psi$. Hence, the choice of observables can strongly influence how models describing a system ought to be coarse grained. For instance, in the original analysis of Feynman and Vernon [14] or Caldeira and Leggett [15], characterizing a particle in a heat bath (albeit in a quantum-mechanical context), the microscopic degrees of freedom contributing to the heat bath were systematically projected away, thereby leaving their effective influence on the particle. Implicitly, in their analysis a choice of $C$ was made and consequently led to the derivation of effective stochastic equations describing the physics of the chosen observables.

The outline of the paper is as follows. In Sec. II we introduce the coarse-graining procedure. We use the procedure in Sec. III to identify the projection operator for diffusion and wave equations. In Sec. IV the projection operators are used to generate $\mathrm{RG}$ flows. We derive the associated $\mathrm{RG}$ equations and contrast the RG flows. Our conclusions are given in Sec. V. Appendixes A and B provide detailed calculations related to the derivation of the projection operators and the $\mathrm{RG}$ equations.

\section{COARSE GRAINING VIA BALANCING}

We coarse grain by retaining the states contributing most to the dynamical response of the system. While technically different, this work is in the same spirit as that by Chen et al. [16] and more recently by Degenhard and Rodríguez-Laguna $[11,17]$. As a starting point, we coarse grain Eq. (4) from its linearization about a particular solution with $u=0$. The reasoning for this approach follows many of the same arguments why linear-response theory has been used with such frequency. Specifically, some of those reasons are that (1) the approach is amenable to calculation, (2) it is often known that the system operates about a particular equilibrium, and (3) for large-amplitude noise, the linear-response dominates since the system then does not get trapped in local equilibria. As a simplification, we only consider linearizations about dynamical steady states. In systems biology, similar approximations are ubiquitously used when describing biochemical networks [12]. The linearizations are generically described by

$$
\begin{gathered}
\dot{\tilde{\varphi}}=A \widetilde{\varphi}+B u, \\
\tilde{\psi}=C \widetilde{\varphi},
\end{gathered}
$$

where $B$ and $C$ are the same operators as those in Eq. (4), $\varphi=\varphi_{e q}+\tilde{\varphi}$ where $\varphi_{e q}$ is an equilibrium solution, and $A$ $=\left.\partial_{\varphi} f\right|_{\varphi_{e q}}$. Associated with Eq. (5) are invariants known as Hankel singular values (HSVs) $[18,19]$. These invariants have many nice properties, but their most significant being 
that they give explicit information about the Green's functions (i.e., dynamical evolution operator) for the system. These invariants are most easily calculated by solving for positive operators $X$ and $Y$ that are determined by the equations

$$
\begin{array}{ll}
\frac{\mathrm{d} X}{\mathrm{~d} t_{f}}=A X+X A^{\dagger}+B B^{\dagger} ; & X(0)=0, \\
\frac{\mathrm{d} Y}{\mathrm{~d} t_{f}}=A^{\dagger} Y+Y A+C^{\dagger} C ; & Y(0)=0 .
\end{array}
$$

If we define another operator $W$ by

$$
W^{2}=X Y,
$$

then the HSV are nonnegative real numbers $\sigma_{\max } \geq \sigma_{\kappa}$ $\geq \sigma_{\text {min }}$ that comprise the spectrum of the operator $W$. HSVs provide a precise measure of the error incurred by approximating the effect $u$ has on $\widetilde{\psi}$ with reduced order models. The HSVs may be interpreted as supplying a measure of the importance of the internal states $\tilde{\varphi}$. If $W$ is invertible, it is always possible to find a coordinate system called balanced coordinates, such that $X=Y=\operatorname{diag}\left(\sigma_{\max }, \ldots, \sigma_{\min }\right)$. When Eq. (5) is transformed to balanced coordinates, the best reductions are those that project out the states corresponding to small HSV. In other words, the ordering of the HSV, at least locally around an equilibrium configuration in phase space, specifies how to coarse grain a system. An in depth treatment of this material may be found in [20]. It is also sometimes possible to "balance" the full nonlinear system [21].

The RG can easily be adapted for HSV-based coarse graining. Operator theoretic approaches to the RG $[7,17,22-24]$ demonstrate that coarse graining in the Wilsonian $\mathrm{RG}$ is equivalent to multiplying operators or states by projection operators. The essence of this work is to use HSVs to identify the projection operator. As before, suppose that $\kappa$ is a vector index that orders the HSVs $\sigma_{\kappa}$ for Eq. (5) from largest to smallest. A generalized Wilsonian RG procedure is obtained by (1) transforming both the initial conditions and the dynamical variables to balanced coordinates,

$$
\hat{\varphi}(\kappa, t)=\int R(\kappa, \mathbf{x}) \varphi(\mathbf{x}, t) \mathrm{d} \mathbf{x},
$$

so that the distribution $P(\phi, t)$ takes the form,

$$
\begin{aligned}
P(\phi, t)= & \int D \phi_{0} D \varphi D \bar{\varphi} \\
& \times \exp \left[-S_{d y n}\left(\mathbf{g},\{\varphi\},\{\bar{\varphi}\},\left\{\phi_{0}\right\}\right)-S\left(\mathbf{g},\left\{\phi_{0}\right\}\right)\right] \\
= & \int D \hat{\phi}_{0} D \hat{\varphi} D \hat{\bar{\varphi}} \mathcal{J} \exp \left[-S_{d y n}\left(\widetilde{\mathbf{g}},\{\hat{\varphi}\},\{\hat{\bar{\varphi}}\},\left\{\hat{\boldsymbol{\phi}}_{0}\right\}\right)\right. \\
& \left.-S\left(\widetilde{\mathbf{g}},\left\{\hat{\phi}_{0}\right\}\right)\right],
\end{aligned}
$$

where $S_{d y n}$ is the action representing the dynamics, $S$ is the action of the initial conditions, $\mathbf{g}$ is the original set of coupling constants/functions, $\bar{\varphi}$ is the dual field that arises from representing the functional $\delta$ in Eq. (2) as an exponential, $\mathcal{J}$ is the Jacobian from Eq. (9), and $\widetilde{\mathbf{g}}$ is the resulting trans- formed set of coupling constants; (2) integrating out $\kappa$ shells about $\sigma_{\min }$ analogously to wave-vector shells; and (3) rescaling $\kappa$ and $\hat{\phi}$ appropriately. An interesting but technically challenging variant of this procedure is to integrate out $\sigma_{\kappa}$ shells instead of $\kappa$ shells about $\sigma_{\text {min }}$. Integrating out a single $\sigma_{\kappa}$ shell may entail integrating out an entire subspace in $\mathbf{x}$ space because $\sigma_{\kappa}$ does not necessarily respect the spatial dimension. The technical challenge lies in rescaling $\sigma_{\kappa}$. It is not clear that rescaling $\sigma_{\kappa}$ will recover the full $\kappa$ space, thereby generating a meaningful RG.

A standard interpretations of the large wave-vector cutoff in the RG is that the inverse cutoff is proportional to the smallest length scale of the system $[7,8]$. The idea of projecting out the large wave-vector physics down to the small wave-vector physics has the interpretation of homogenizing the system to its continuum large-scale limit. However, interfaces that are smooth on short spatial scales may actually appear sharp when viewed on large scales. Representing such effective sharp interfaces in terms of Fourier (wavevector) modes then requires a huge number of modes (the largest mode being the cutoff). Other representations, such as wavelet representations, are much better equipped to describe the singular nature of such interfaces. Mathematically, this means that the cutoff is specifically chosen because it provides us with a starting point for coarse graining and an ordering relation that determines in which direction to coarse grain. The cutoff can be thought of entirely in approximation/interpolation theoretic terms. The initiation of coarse graining starts with modes contributing the least to the phenomena of interest. The ordering relation results from the ordering of modes by their contribution to the approximation. $\sigma_{\min }$ is a generalization of the standard wave-vector cutoff and the ordering relation determined by the ordering of the HSVs $\left(\sigma_{\kappa}\right)$ is the generalization of the standard scale ordering (small scale to large scale). The RG has been studied in the context of approximation/interpolation theory before. RG methods have been related to theory of Padé approximants by Baker and Graves-Morris [25], to wavelet theory by Battle [26,27], and to subspace/Lanzcos methods by White [28,29].

\section{IDENTIFICATION OF THE PROJECTION OPERATOR}

Here we apply the aforementioned coarse-graining procedure to identify the projection operator for linear diffusion and linear and nonlinear wave equations. Since the procedure requires us to expand about a dynamical steady state, if we expand about the trivial solution, then the nonlinearity does not change anything. In this section, we find that by considering slow observables for the diffusion equation and wave equation, projecting out large wave-vector shells is appropriate. When we additionally consider the fast kinetic observables for the wave equation, we arrive at quite a different result. This results from the extra time derivative of the wave equation and is the very reason why we consider the NLWE in this paper.

\section{A. Linear diffusion equation}

We first consider the (driven) diffusion equation 


$$
\partial_{t} \phi=D \nabla^{2} \phi+\gamma u \text {. }
$$

In this example, $B=\gamma, C=1, A=D \nabla^{2}$, and we take $t_{f} \rightarrow \infty$. By considering a stable system over an infinite time horizon, we only need to solve the Lyapunov equations,

$$
\begin{aligned}
& A X+X A^{\dagger}+B B^{\dagger}=0, \\
& A^{\dagger} Y+Y A+C^{\dagger} C=0,
\end{aligned}
$$

instead of Eqs. (6) and (7). By taking the Fourier transform, Eqs. (12) and (13) become

$$
\begin{gathered}
2 D|\mathbf{k}|^{2} X_{\mathbf{k}}+|\gamma|^{2}=0, \\
2 D|\mathbf{k}|^{2} Y_{\mathbf{k}}+1=0 .
\end{gathered}
$$

From Eq. (8), aside from some subtle technical issues arising when $|\mathbf{k}|=0$ [30], it follows that in balanced coordinates $W$ is given by

$$
W_{\mathbf{k}}=\frac{|\gamma|}{2 D|\mathbf{k}|^{2}} .
$$

Here the index $\kappa$ for the HSVs is just $|\mathbf{k}|$. Thus, for the diffusion equation, the most important states are those that correspond to small wave vector. Thus, local coarse graining is appropriate because the smallest observable "fluctuations" are due to the short-wavelength physics. The smallest error is incurred by projecting out large wave vectors. We have thus identified the projection operator for the diffusion equation and it exactly coincides with what we would expect. Diffusive dynamics spatially homogenizes disturbances, thus, intuitively we already know that local coarse graining is desirable.

\section{B. Linear wave equation}

As a second example, we consider the (driven) linear wave equation

$$
\begin{gathered}
\partial_{t}^{2} \phi=v^{2} \nabla^{2} \phi+\gamma u, \\
\mathbf{y}=\phi .
\end{gathered}
$$

By the units of $u$, it represents a true force acting on $\phi$. This, in addition to the fact that $\phi$ is the "measurable" quantity, implies that we have isolated our attention on $\phi$-based observables. This choice is strongly influenced by equilibrium statistical mechanics and thermodynamics. We have completely neglected $\pi$ (the field conjugate to $\phi$ ) that represents the kinetic contributions to the system. When posed as a set of first-order equations, Eq. (17) becomes

$$
\left[\begin{array}{c}
\partial_{t} \phi \\
\partial_{t} \boldsymbol{\pi} \\
\mathbf{y}
\end{array}\right]=\left[\begin{array}{ccc}
0 & 1 & 0 \\
v^{2} \nabla^{2} & 0 & \gamma \\
1 & 0 & 0
\end{array}\right]\left[\begin{array}{l}
\phi \\
\pi \\
u
\end{array}\right] .
$$

By smoothing out the time cutoff at $t_{f}$ with a damped exponential in the integral representation of the solution of Eqs. (6) and (7), the problem simplifies to solving Lyapunov equations. This smoothing process is also known as expo- nential discounting. With the given form of $B$ and $C$ in this problem, we find that the matrix of HSVs $(W)$ is approximately given by

$$
W_{\mathbf{k}}^{b a l} \approx \frac{|\gamma|}{4 a v|\mathbf{k}|} \otimes I_{2 \times 2},
$$

where $I_{2 \times 2}$ is the $2 \times 2$ matrix identity, $a \sim 1 / t_{f}$, and $\otimes$ is the tensor (dyadic) product. The calculation that produces this result is a special case of the calculation in Appendix A. As with the diffusion equation, short-wavelength physics does not significantly contribute to the response, so locally coarse graining is appropriate. In the examples considered here, the dynamical equations that specify the projection operator are linear. Thus, no approximation has been made and we expect that the corresponding projection operators are globally valid in phase space.

\section{Nonlinear wave equation with nonequilibrium observables}

We now consider a NLWE with a nonequilibrium set of observables. As will be seen, a surprising result is that the choice of observables forces us to nonlocally coarse grain. The nonlocality of the coarse graining has very interesting implications with regard to the resulting induced $\mathrm{RG}$ flow. The (driven) equations of motion that we are considering are

$$
\begin{gathered}
\partial_{t} \phi=\pi+\alpha_{1} u_{1}, \\
\partial_{t} \pi=\nabla^{2} \phi+\frac{\lambda}{3 !} \phi^{3}+\alpha_{2} u_{2}, \\
\mathbf{y}=\left[\begin{array}{l}
\beta_{1} \phi \\
\beta_{2} \pi
\end{array}\right],
\end{gathered}
$$

where $\phi$ and $\pi$ are real-valued fields. The driving now includes generalized forces in addition to "true" forces. By expanding around equilibrium solutions of $\nabla^{2} \phi=0$, we find that for each real-space position $\mathbf{x}$,

$$
\begin{aligned}
& B=\left(\begin{array}{cc}
\alpha_{1} & 0 \\
0 & \alpha_{2}
\end{array}\right), \\
& C=\left(\begin{array}{cc}
\beta_{1} & 0 \\
0 & \beta_{2}
\end{array}\right) .
\end{aligned}
$$

This driving allows for more states in $(\phi, \pi)$-phase space to be accessible compared to the driving in Eq. (18). This, in combination with the form of $\mathbf{y}$, ensures that both $\phi$ - and $\pi$-dependent observables are being considered. By using exponential discounting, we find that the diagonal operator of HSVs is given by (see Appendix A)

$$
W_{\mathbf{k}} \approx \frac{1}{4 a}\left[\left(\alpha_{2}^{2}|\mathbf{k}|^{-1}+\alpha_{1}^{2}|\mathbf{k}|\right)\left(\beta_{1}^{2}|\mathbf{k}|^{-1}+\beta_{2}^{2}|\mathbf{k}|\right)\right]^{1 / 2} \otimes I_{2 \times 2} .
$$

$W_{\mathbf{k}}$ does not have the HSVs ordered from largest to smallest, so it is not truly in balanced coordinates. It is immediately apparent that the HSVs are large for both large and small 
magnitude wave vectors. A heuristic explanation for this strange result is that for large wave vector, $\pi$ is a pathologically "fast" variable. However, by driving $\pi$ with $u_{1}$ over all wave vector, this permits the fast resonances to be excited at large wave vector. The pathological nature of $\pi$ as an observable is analogous to the pathological nature of considering $\dot{\xi}$ an observable where $\xi$ satisfies a Langevin equation [31]. In this sense, $\pi$ is a nonequilibrium observable of sorts. Furthermore, because both the small and large wavelength physics contribute strongly to the response of the system, local coarse graining cannot be correct. The appropriate coarse graining is nonlocal.

\section{RG ANALYSIS}

Now that we have specified how to coarse grain, we must specify the distribution of initial conditions that we wish to coarse grain. Should we choose a Gaussian distribution of initial conditions then it turns out that provided that the action $S\left(\mathbf{g},\left\{\phi_{0}\right\}\right)$ does not have a nonlocal spatial dependence, then the integration regions decouple in Fourier space and the $\mathrm{RG}$ is trivial. We thus consider a distribution of initial conditions that is local and quartic. Specifically, we consider the action for the initial conditions to be

$$
S\left(\mathbf{g},\left\{\phi_{0}\right\}\right)=\int \mathrm{d} \mathbf{x}\left(\frac{1}{2} \nabla \phi_{0} \nabla \phi_{0}+\frac{\lambda}{4 !} \phi_{0}^{4}\right) .
$$

It is important to remark here that we consider the gradient term to be local (or marginally local). Equivalently, in Fourier space, the action is given by

$$
\begin{aligned}
S\left(\mathbf{g},\left\{\hat{\phi}_{0}\right\}\right)= & \frac{1}{2(2 \pi)^{D}} \int \mathrm{d} \mathbf{k}|\mathbf{k}|^{2}\left|\hat{\phi}_{0}(\mathbf{k})\right|^{2} \\
& +\frac{\lambda}{4 !} \int \prod_{n=1}^{4} \frac{\mathrm{d} \mathbf{k}_{n}}{(2 \pi)^{D}} \delta\left(\sum_{j=1}^{4} \mathbf{k}_{j}\right) \\
& \times \hat{\phi}_{0}\left(\mathbf{k}_{1}\right) \hat{\phi}_{0}\left(\mathbf{k}_{2}\right) \hat{\phi}_{0}\left(\mathbf{k}_{3}\right) \hat{\phi}_{0}\left(\mathbf{k}_{4}\right),
\end{aligned}
$$

where $D$ is the spatial dimension of the system. Rather than being wholly unmotivated, with the addition of kinetic terms, the CTP method $[4,5]$ may be used to derive a dynamical action from the above $S$ that is approximately the same as $S_{d y n}$ in Eq. (10). Also, in the remainder, to avoid unnecessary subscripts, we denote $\hat{\phi}_{0}$ by $\hat{\phi}$ with the understanding that we are performing the RG on the distribution of initial conditions.

\section{A. RG equations from local coarse graining}

Here we introduce a large wave-vector cutoff $\Omega$. We define $\hat{\phi}=\hat{\phi}_{<}+\hat{\phi}_{>}$, where $\hat{\phi}_{<}$is only nonzero for $|\mathbf{k}| \leq \Omega / b$ $(b>1)$ and $\hat{\phi}_{>}$is only nonzero for $\Omega / b<|\mathbf{k}|<\Omega$. Integrating out wave-vector shells between $\Omega / b$ and $\Omega$ entails integrating out the $\hat{\phi}_{>}$fields. We then rescale by defining

$$
\hat{\phi}_{<}(\mathbf{k})=Z \varphi_{1}(b \mathbf{k})
$$

and $\mathbf{p}=b \mathbf{k}$. Although we do not start with a "mass" term in the action (i.e., $m^{2} \phi^{2}$ ), such a term is generated by the RG flow. Upon following the Wilson RG variant of what is described in Appendix B or the analysis done by Shankar in [10], we obtain the RG equations

$$
\begin{gathered}
\partial_{l} \bar{\lambda}=(4-D) \bar{\lambda}-\frac{3}{2} \frac{S_{D}}{(2 \pi)^{D}} \bar{\lambda}^{2}+\mathcal{O}\left(\bar{\lambda}^{3}\right), \\
\partial_{l} \bar{m}^{2}=2 \bar{m}^{2}+\frac{S_{D}}{2(2 \pi)^{D}} \bar{\lambda}\left(1-\bar{m}^{2}\right)+\mathcal{O}\left(\bar{\lambda}^{2}\right) .
\end{gathered}
$$

$\bar{\lambda}$ and $\bar{m}^{2}$ are dimensionless and result from appropriately rescaling $\lambda$ and $m^{2}$ by $\Omega$. The RG flow and fixed points for these equations are well studied. Two fixed points are the Gaussian fixed point $\bar{m}^{2}=0$ and $\bar{\lambda}=0$ and the Wilson-Fisher fixed point $\bar{m}^{2} \approx-(4-D) / 6$ and $\bar{\lambda} \approx 2(4-D)(2 \pi)^{D} /\left(3 S_{D}\right)$. Following standard convention, we approximate the WilsonFisher fixed point in powers of $4-D$ (the $\epsilon$ expansion). We provide the form of these $\mathrm{RG}$ equations because, as will soon be evident, they differ greatly from those that we derive in the next section.

\section{B. RG equations from nonlocal coarse graining}

In the case where $\phi$ and $\pi$ are treated on equal footing as observables, which in general may not be the case, $\alpha_{1}=\alpha_{2}$ $=\alpha$ and $\beta_{1}=\beta_{2}=\beta$. In the remainder, we treat this particular case. Furthermore, without loss of generality, we set $\alpha=\beta$ $=1$. In this case, Eq. (23) indicates that the $|\mathbf{k}|=1$ states are the least important. Thus, for the purposes of the RG, the $|\mathbf{k}|=1$ hypersurface serves as our analog of the wave-vector cutoff. Implementing the second step of the procedure for the generalized RG involves integrating out $\mathbf{k}$ shells away from the $|\mathbf{k}|=1$ surface. Rather than transform the system into the balanced $\kappa$ coordinates, out of convenience, we coarse grain the system in $\mathbf{k}$ space.

In order to coarse grain, we let $\hat{\phi}=\hat{\phi}_{<}+\hat{\phi}_{m}+\hat{\phi}_{>}$where $\hat{\phi}_{<}$is only nonzero for $|\mathbf{k}| \leq \Lambda, \hat{\phi}_{m}$ is only nonzero for $\Lambda$ $<|\mathbf{k}|<\Lambda^{-1}$, and $\hat{\phi}_{>}$is only nonzero for $|\mathbf{k}| \geq \Lambda^{-1}$, where $\Lambda$ $<1$. Using this decomposition, the path-integral measure factors as $\mathcal{D} \hat{\phi}=\mathcal{D} \hat{\phi}_{<} \mathcal{D} \hat{\phi}_{m} \mathcal{D} \hat{\phi}_{>}$. The $\mathrm{RG}$ equations are then induced by integrating out $\hat{\phi}_{m}$ and then rescaling the wave vectors and fields. For this problem, the rescaling procedure requires that

$$
\begin{gathered}
\hat{\phi}_{<}(\mathbf{k})=Z_{<} \varphi_{1}\left(\Lambda^{-1} \mathbf{k}\right), \\
\hat{\phi}_{>}(\mathbf{k})=Z_{>} \varphi_{2}(\Lambda \mathbf{k}),
\end{gathered}
$$

and $\mathbf{p}=\Lambda^{-1} \mathbf{k}$ for $|\mathbf{k}| \leq \Lambda$ and $\mathbf{p}=\Lambda \mathbf{k}$ for $|\mathbf{k}| \geq \Lambda^{-1}$. Naïve power counting breaks down as a direct result of rescaling in the two disjoint wave-vector regimes.

Although we start with a theory where $\mathbf{g}=(1, \lambda, 0, \ldots)$, we can expect that the RG transformations may generate new nonlinear terms and that the coupling constants may become coupling functions. In fact, $\mathbf{g}$ flows toward having an infinite number of nontrivial components. In particular, in a complete treatment, the coupling constant $\lambda$ becomes a coupling function $\lambda\left(\mathbf{p}_{1}, \mathbf{p}_{2}, \mathbf{p}_{3}, \mathbf{p}_{4}\right)$. However, if we focus purely on the 
TABLE I. Relevance of perturbations when $D=4$.

\begin{tabular}{lcccccc}
\hline \hline & $|p|<1$ & & & \\
\hline Coarse & $p^{n} \hat{\phi}^{2}$, & $p^{n} \hat{\phi}^{m}, n>0$, & $\hat{\phi}^{n}$, & $p^{n} \hat{\phi}^{2}$, & $p^{n} \hat{\phi}^{m}, n>0$, & $\hat{\phi}^{n}$, \\
graining & $n>2$ & $m \geq 4$ & $n>4$ & $n>2$ & $m \geq 4$ & $n>4$ \\
Local & no & no & no & no & no & no \\
Nonlocal & no & no & no & yes & yes & yes \\
\hline \hline
\end{tabular}

constant contributions of $\lambda\left(\mathbf{p}_{1}, \mathbf{p}_{2}, \mathbf{p}_{3}, \mathbf{p}_{4}\right)$ in the different wave-vector scaling regimes, we see that it may be decomposed into the couplings $\left\{\lambda_{(i, 4-i)}\right\}_{i=1}^{4}$. Here $\lambda_{(i, j)}$ represents the coupling constant in the scaling regime with $i$ wave vectors having $\left|\mathbf{p}_{n}\right|<1$ and $j$ wave vectors having $\left|\mathbf{p}_{n}\right|>1$. We denote the mass terms for $|\mathbf{q}|<1$ and $|\mathbf{q}|>1$, respectively, by $m_{<}^{2}$ and $m_{>}^{2}$. If we let $\Lambda=e^{-d l}$ then to the first loop order the $\mathrm{RG}$ equations for $\left\{\lambda_{(i, 4-i)}\right\}_{i=1}^{4}$ are

$$
\begin{aligned}
\partial_{l} \lambda_{(4,0)}= & (4-D) \lambda_{(4,0)}-\frac{3}{2} \frac{S_{D}}{(2 \pi)^{D}}\left[\lambda_{(4,0)}^{2}\left(m_{<}^{2}+1\right)^{-2}\right. \\
& \left.+2 \lambda_{(3,1)}^{2}\left(m_{<}^{2}+1\right)^{-1}\left(m_{>}^{2}+1\right)^{-1}+\lambda_{(2,2)}^{2}\left(m_{>}^{2}+1\right)^{-2}\right],
\end{aligned}
$$

$$
\begin{aligned}
\partial_{l} \lambda_{(0,4)}= & (D-4) \lambda_{(0,4)}-\frac{3}{2} \frac{S_{D}}{(2 \pi)^{D}}\left[\lambda_{(2,2)}^{2}\left(m_{<}^{2}+1\right)^{-2}\right. \\
& \left.+2 \lambda_{(1,3)}^{2}\left(m_{<}^{2}+1\right)^{-1}\left(m_{>}^{2}+1\right)^{-1}+\lambda_{(0,4)}^{2}\left(m_{>}^{2}+1\right)^{-2}\right],
\end{aligned}
$$

$$
\begin{aligned}
\partial_{l} \lambda_{(1,3)}= & -2 \lambda_{(1,3)}-6 \frac{S_{D}}{(2 \pi)^{D}}\left[\lambda_{(2,2)} \lambda_{(3,1)}\left(m_{<}^{2}+1\right)^{-2}\right. \\
& +2 \lambda_{(1,3)} \lambda_{(2,2)}\left(m_{<}^{2}+1\right)^{-1}\left(m_{>}^{2}+1\right)^{-1} \\
& \left.+\lambda_{(0,4)} \lambda_{(1,3)}\left(m_{>}^{2}+1\right)^{-2}\right] \\
\partial_{l} \lambda_{(3,1)}= & -2(D-1) \lambda_{(3,1)}-6 \frac{S_{D}}{(2 \pi)^{D}}\left[\lambda_{(4,0)} \lambda_{(3,1)}\left(m_{<}^{2}+1\right)^{-2}\right. \\
+ & 2 \lambda_{(3,1)} \lambda_{(2,2)}\left(m_{<}^{2}+1\right)^{-1}\left(m_{>}^{2}+1\right)^{-1} \\
+ & \left.\lambda_{(2,2)} \lambda_{(1,3)}\left(m_{>}^{2}+1\right)^{-2}\right]
\end{aligned}
$$

$$
\begin{aligned}
\partial_{l} \lambda_{(2,2)}= & -D \lambda_{(2,2)}-3 \frac{S_{D}}{(2 \pi)^{D}}\left[\left(\lambda_{(2,2)} \lambda_{(4,0)}+2 \lambda_{(3,1)}^{2}\right)\left(m_{<}^{2}+1\right)^{-2}\right. \\
& +2\left(\lambda_{(1,3)} \lambda_{(3,1)}+2 \lambda_{(2,2)}^{2}\right)\left(m_{<}^{2}+1\right)^{-1}\left(m_{>}^{2}+1\right)^{-1} \\
& \left.+\left(\lambda_{(0,4)} \lambda_{(2,2)}+2 \lambda_{(1,3)}^{2}\right)\left(m_{>}^{2}+1\right)^{-2}\right] .
\end{aligned}
$$

The RG equations for the mass terms are

$$
\partial_{l} m_{<}^{2}=2 m_{<}^{2}+\frac{S_{D}}{2(2 \pi)^{D}}\left(\frac{\lambda_{(4,0)}}{m_{<}^{2}+1}+\frac{\lambda_{(2,2)}}{m_{>}^{2}+1}\right)+\mathcal{O}\left(\lambda^{2}\right),
$$

$$
\partial_{l} m_{>}^{2}=-2 m_{>}^{2}+\frac{S_{D}}{2(2 \pi)^{D}}\left(\frac{\lambda_{(2,2)}}{m_{<}^{2}+1}+\frac{\lambda_{(0,4)}}{m_{>}^{2}+1}\right)+\mathcal{O}\left(\lambda^{2}\right) .
$$

The first thing to notice in Eqs. (30)-(34) is that the contributions from tree level (the linear terms) indicate that the couplings involving a mixing of wave vectors (i.e., $i, j \neq 0$ ) are irrelevant. The terms that are relevant appear similar to Eqs. (26) and (27). In fact, if we expand about $m_{<}^{2}=0$, make the identifications $\lambda_{(4,0)}=\bar{\lambda}$ and $m_{<}^{2}=\bar{m}^{2}$, and set the rest of the coupling constants to zero, then Eqs. (30) and (35) are exactly the same as Eqs. (26) and (27). Thus, if it were possible to ignore all other relevant perturbations, then the new set of RG equations would retain the standard fixed points (Gaussian and Wilson Fisher). With local coarse graining, higher-order derivatives and nonlinearities are rendered irrelevant by coarse graining. This then justifies why we only need to renormalize the $\bar{m}^{2}$ and $\bar{\lambda}$ couplings. However, upon nonlocally coarse graining the system, it is no longer possible to ignore the wave-vector dependence that $\lambda$ acquires. Such perturbations are relevant for $\left|\mathbf{p}_{i}\right|>1$. Specifically, higher derivative perturbations $p^{n} \hat{\phi}^{2}, n>2$ and $p^{n} \hat{\phi}^{m}, n>0, m \geq 4$ in addition to higher-order nonlinearities $\hat{\phi}^{n}, n>4$ become relevant when $\left|\mathbf{p}_{i}\right|>1$. While the couplings at small wave vector $\left|\mathbf{p}_{i}\right|<1$ almost obey the standard RG equations obtained by local coarse graining, if the system is perturbed to its large wave-vector regime, then the couplings will flow away from the Gaussian or Wilson-Fisher fixed points [10]. This reflects that the dynamically faster shortwavelength perturbations are able to excite the conjugate field $\pi$, thereby driving the system away from its standard statistical equilibrium. Were the conjugate field not accessible to the "noise" $\alpha_{1}=0$ or not an observable $\beta_{2}=0$, this would not have occurred. We summarize the relevancy of perturbations in Table I in the case when $D=4$. A key observation to make from our analysis is that the nonlocal coarsegraining produces $\mathrm{RG}$ equations that are very different from the canonical ones [Eqs. (26) and (27)]. In fact, a complete analysis would require us to determine the RG flow of infinitely many coupling constants.

While the existence of an infinity of relevant perturbations renders Eqs. (30)-(36) meaningless, for completeness, we present their fixed-point structure anyhow. One fixed point of the equations exists where $m_{<}^{2} \approx-(4-D) / 6$, $\lambda_{(4,0)} \approx 2(4-D)(2 \pi)^{D /}\left(3 S_{D}\right), \quad m_{>}^{2} \approx(4-D) / 6, \quad \lambda_{(0,4)} \approx-2(4$ $-D)(2 \pi)^{D} /\left(3 S_{D}\right)$, and the rest of the couplings zero. A problem with it is that it does not guarantee positivity of the 
action. This already suggests the need to include higher-order relevant terms. For instance, to fix the positivity issue, we would need to add at least a $\hat{\phi}^{6}$ term to the action. Similarly, for $D \leq 4$, the irrelevancy of the rest of the $\lambda_{(i, j)}$ terms ensures that we would lose positivity, thereby requiring the addition of higher-order terms. However, for $D>4$, there is an alternative fixed point that guarantees positivity of the action. At this fixed point, the couplings are $m_{>}^{2} \approx(4-D) / 6$, $\lambda_{(0,4)} \approx-2(4-D)(2 \pi)^{D} /\left(3 S_{D}\right)$, and the rest zero. While this fixed point seems strikingly similar to the Wilson-Fisher fixed point, it is important to recognize that the coupling constant multiplies products of fields in a different wavevector regime.

Although we have already compared our results to those obtained by canonically coarse graining the same distribution of initial conditions in Sec. IV A, we have yet to relate them to other work in the NLWE literature. The RG has been applied to NLWEs by many authors in a variety of different contexts, two examples of which are [32,33]. In [32], Matsuba and Nozaki applied a perturbative RG method to a weakly nonlinear wave equation in order to derive an effective amplitude equation. The effective amplitude equation that they derive is the nonlinear Schrödinger equation. In quite a different context, Bricmont et al. [33] applied the RG to a NLWE in order to prove the persistence of quasiperiodic low-dimensional elliptic tori. In contrast, we derive the projection operator from the dynamics of the NLWE but do not apply the RG to the dynamical equations. Instead, we apply the RG to the distribution of initial conditions.

Complementary research that applies projection-operator methods to wave equations includes the work of Boesch et al. [34], Brun and Hartle [35], and Maïzi [36]. Boesch et al. used projection-operator techniques to derive equations of motion for collective coordinates describing solitonlike solutions to nonlinear Klein-Gordon equations. They found that projection-operator techniques greatly facilitate the derivation of the aforementioned equations of motion. While there are many differences, a key distinction between our work and that of Boesch et al. is that we do not a priori select our observables. In [35], Brun and Hartle investigated the coherence of coarse-grained trajectories of the homogeneous quantum harmonic chain. The coherence of the trajectories reflects the classical behavior of the dynamics. After assuming a particular form of the initial conditions and investigating a class of possible coarse grainings, they concluded that the decoherence time for coarse-grained trajectories increases with the locality of the coarse graining. As should be expected, when applied to the quantum harmonic chain, it is a simple exercise to show that HSV coarse graining allows one to derive the result of Brun and Hartle. Lastly, in [36], Maïzi applied HSV analysis to a one-dimensional wave equation subject to boundary forcing. Maïzi found that $\sigma_{k}$ $\sim 1 / a|k|$, that is, similar to what we find in Eq. (19) in the case of bulk forcing.

\section{CONCLUSION}

In this paper, we have presented a RG procedure and have applied it to a nonlinear wave equation with probabilistic initial conditions. We have shown that when both equilibrium and nonequilibrium observables are chosen, this $\mathrm{RG}$ procedure predicts that naïve power counting breaks down and that terms that are ordinarily irrelevant become relevant at large wave vector. We have shown that the RG equations that we have derived using nonlocal coarse graining differ significantly from the RG-equations-derived local coarse graining. While our RG equations superficially retain the Gaussian and Wilson-Fisher fixed points as solutions, the infinity of relevant perturbations ensures that such fixed points either do not exist or that they pick up an infinity of unstable directions in the RG flow. Additionally, the equations superficially admit many other fixed points. The generalized Wilsonian RG developed herein is applicable to any system where it is desirable to coarse grain based on the intrinsic system dynamics. Although the RG is still formally an uncontrolled approximation, the coarse graining is chosen such that the effective coarsened system is close to the original one. Despite the versatility in this method, it is often difficult to analytically determine the balancing transformations. However, since there are very efficient numerical algorithms for finding balanced coordinates, this generalized RG remains a numerically useful and practical algorithm. Lastly, in the context of studying complex uncertain biochemical networks, we have found that the projection operators we derive are exceptionally useful but are more easily used in one-step coarse graining than in iterative coarse graining $(\mathrm{RG})$.

\section{ACKNOWLEDGMENTS}

This work was supported by the NSF under Grant No. DMR-9813752 and the NSF FIBR under Grant No. 492153. Special thanks are due to Jean Carlson for her comments and to Nigel Goldenfeld for his comments and encouragement.

\section{APPENDIX A: BALANCING $X$ AND $Y$}

In this appendix, we intend to calculate the balanced form of the operators $X$ and $Y$ [from Eqs. (6) and (7)] called gramians for linear wave equations (or their discretizations). This entails calculating the damped (exponentially discounted) gramians,

$$
\begin{aligned}
& X^{(a)}=\int_{0}^{\infty} e^{-2 a t} e^{A t} B B^{\dagger} e^{A^{\dagger} t} \mathrm{~d} t, \\
& Y^{(a)}=\int_{0}^{\infty} e^{-2 a t} e^{A^{\dagger} t} C^{\dagger} C e^{A t} \mathrm{~d} t .
\end{aligned}
$$

As in the body of the paper,

$$
\begin{aligned}
& B=\left(\begin{array}{cc}
\alpha_{1} \mathbf{I} & 0 \\
0 & \alpha_{2} \mathbf{I}
\end{array}\right), \\
& C=\left(\begin{array}{cc}
\beta_{1} \mathbf{I} & 0 \\
0 & \beta_{2} \mathbf{I}
\end{array}\right) .
\end{aligned}
$$


Let us first introduce the following notations and conventions. Recall that any matrix $\mathbf{S}$ may be expressed in terms of the canonical matrix units $\mathbf{e}_{i j}$. In other words,

$$
\mathbf{S}=\sum_{i, j} S_{i j} \mathbf{e}_{i j},
$$

where each $S_{i j}$ is just a complex number. For instance, in the case of $2 \times 2$ matrices,

$$
\mathbf{e}_{12}=\left[\begin{array}{ll}
0 & 1 \\
0 & 0
\end{array}\right] .
$$

Additionally, for this section, $\mathbf{Q}=\left[\begin{array}{cc}0 & -1 \\ 1 & 0\end{array}\right]$. Lastly, we frequently use the algebraic tensor (dyadic) product $\otimes$. For instance, suppose

$$
A=\left[\begin{array}{ll}
A_{11} & A_{12} \\
A_{21} & A_{22}
\end{array}\right]
$$

then

$$
A \otimes B=\left[\begin{array}{ll}
A_{11} B & A_{12} B \\
A_{21} B & A_{22} B
\end{array}\right]
$$

$$
\begin{aligned}
& X^{(a)}=\mathbf{R} \int_{0}^{\infty} e^{-2 a t}\left[\begin{array}{cc}
\alpha_{1}^{2} \boldsymbol{\Omega} \cos ^{2} \boldsymbol{\Omega} t+\alpha_{2}^{2} \boldsymbol{\Omega}^{-1} \sin ^{2} \boldsymbol{\Omega} t & \frac{1}{2} \boldsymbol{\Omega}^{-1}\left(\alpha_{2}^{2} \boldsymbol{\Omega}^{-1}-\alpha_{1}^{2} \boldsymbol{\Omega}\right) \frac{\mathrm{d}}{\mathrm{d} t} \sin ^{2} \boldsymbol{\Omega} t \\
\frac{1}{2} \boldsymbol{\Omega}^{-1}\left(\alpha_{2}^{2} \boldsymbol{\Omega}^{-1}-\alpha_{1}^{2} \boldsymbol{\Omega}\right) \frac{\mathrm{d}}{\mathrm{d} t} \sin ^{2} \boldsymbol{\Omega} t & \alpha_{2}^{2} \boldsymbol{\Omega}^{-1} \cos ^{2} \boldsymbol{\Omega} t+\alpha_{1}^{2} \boldsymbol{\Omega} \sin ^{2} \boldsymbol{\Omega} t
\end{array}\right] \mathrm{d} t \mathbf{R} \\
& =\frac{1}{4 a} \mathbf{R}\left[\begin{array}{cc}
\alpha_{1}^{2} \boldsymbol{\Omega}+\alpha_{2}^{2} \mathbf{\Omega}^{-1} & 0 \\
0 & \alpha_{1}^{2} \boldsymbol{\Omega}+\alpha_{2}^{2} \mathbf{\Omega}^{-1}
\end{array}\right] \mathbf{R} \\
& +\frac{1}{4 a} \mathbf{R}\left[\begin{array}{cc}
-a^{2} \boldsymbol{\Omega}^{-1}\left(\alpha_{2}^{2} \mathbf{I}-\alpha_{1}^{2} \mathbf{\Omega}^{2}\right)\left(a^{2} \mathbf{I}+\boldsymbol{\Omega}^{2}\right)^{-1} & a\left(\alpha_{2}^{2} \mathbf{I}-\alpha_{1}^{2} \boldsymbol{\Omega}^{2}\right)\left(a^{2} \mathbf{I}+\mathbf{\Omega}^{2}\right)^{-1} \\
a\left(\alpha_{2}^{2} \mathbf{I}-\alpha_{1}^{2} \boldsymbol{\Omega}^{2}\right)\left(a^{2} \mathbf{I}+\boldsymbol{\Omega}^{2}\right)^{-1} & a^{2} \boldsymbol{\Omega}^{-1}\left(\alpha_{2}^{2} \mathbf{I}-\alpha_{1}^{2} \mathbf{\Omega}^{2}\right)\left(a^{2} \mathbf{I}+\mathbf{\Omega}^{2}\right)^{-1}
\end{array}\right] \mathbf{R} .
\end{aligned}
$$

Similarly for the other gramian, we obtain

$$
\begin{aligned}
Y^{(a)}= & \frac{1}{4 a} \mathbf{R}^{-1}\left[\begin{array}{cc}
\beta_{2}^{2} \boldsymbol{\Omega}+\beta_{1}^{2} \mathbf{\Omega}^{-1} & 0 \\
0 & \beta_{2}^{2} \boldsymbol{\Omega}+\beta_{1}^{2} \mathbf{\Omega}^{-1}
\end{array}\right] \mathbf{R}^{-1} \\
& +\frac{1}{4 a} \mathbf{R}^{-1}\left[\begin{array}{cc}
a^{2} \mathbf{\Omega}^{-1}\left(\beta_{1}^{2} \mathbf{I}-\beta_{2}^{2} \boldsymbol{\Omega}^{2}\right)\left(a^{2} \mathbf{I}+\boldsymbol{\Omega}^{2}\right)^{-1} & a\left(\beta_{1}^{2} \mathbf{I}-\beta_{2}^{2} \mathbf{\Omega}^{2}\right)\left(a^{2} \mathbf{I}+\boldsymbol{\Omega}^{2}\right)^{-1} \\
a\left(\beta_{1}^{2} \mathbf{I}-\beta_{2}^{2} \mathbf{\Omega}^{2}\right)\left(a^{2} \mathbf{I}+\mathbf{\Omega}^{2}\right)^{-1} & -a^{2} \mathbf{\Omega}^{-1}\left(\beta_{1}^{2} \mathbf{I}-\beta_{2}^{2} \mathbf{\Omega}^{2}\right)\left(a^{2} \mathbf{I}+\mathbf{\Omega}^{2}\right)^{-1}
\end{array}\right] \mathbf{R}^{-1} .
\end{aligned}
$$

From Eqs. (A5) and (A6) it follows after using $\mathbf{U}_{d}$ to diagonalize $\boldsymbol{\Omega}$ and taking the small $a$ limit that the balanced gramian, without ordered eigenvalues, is given by

$$
W^{b a l} \approx \frac{1}{4 a}\left[\begin{array}{cc}
\alpha_{1}^{2} \boldsymbol{\Lambda}_{\Omega}+\alpha_{2}^{2} \boldsymbol{\Lambda}_{\Omega}^{-1} & 0 \\
0 & \alpha_{1}^{2} \boldsymbol{\Lambda}_{\Omega}+\alpha_{2}^{2} \boldsymbol{\Lambda}_{\Omega}^{-1}
\end{array}\right]^{1 / 2}\left[\begin{array}{cc}
\beta_{2}^{2} \boldsymbol{\Lambda}_{\Omega}+\beta_{1}^{2} \boldsymbol{\Lambda}_{\Omega}^{-1} & 0 \\
0 & \beta_{2}^{2} \boldsymbol{\Lambda}_{\Omega}+\beta_{1}^{2} \boldsymbol{\Lambda}_{\Omega}^{-1}
\end{array}\right]^{1 / 2} .
$$

\section{APPENDIX B: RG EQUATIONS TO ONE LOOP}

In this appendix, we briefly derive the RG equations for the two-point [Eqs. (35) and (36)] and the four-point coupling functions [Eq. (30)]. In Appendix B, Sec. B 1, we sketch out the standard perturbative procedure [7-10] used to derive the RG equations. Then we proceed to use the perturbative procedure to derive the RG equations for the two- point and four-point couplings in Appendix B, Secs. B 2 and B 3, respectively.

\section{Sketch of perturbation theory}

Recall that we will be integrating out the field variables $\hat{\phi}_{m}$ and keeping $\hat{\phi}_{<}$and $\hat{\phi}_{>}$. Ideally, we would like to exactly evaluate the partial trace 


$$
\int \mathcal{D} \hat{\phi}_{m} \exp \left\{-S\left(\hat{\phi}_{<}, \hat{\phi}_{>}, \hat{\phi}_{m}\right)\right\}=\exp \left\{-\bar{S}\left(\hat{\phi}_{<}, \hat{\phi}_{>},\right)\right\}
$$

In general, computing such a functional integral is very difficult. Consequently, we will perturbatively evaluate Eq. (B1) about the quadratic part of the action. Now let us denote the quadratic part of the action by $S_{2}$ and the remainder by $S_{r}$ (i.e., $S=S_{2}+S_{r}$ ). By the simple form of the quadratic part of the action in Eq. (24), we have that

$$
S_{2}\left(\hat{\phi}_{<}, \hat{\phi}_{>}, \hat{\phi}_{m}\right)=S_{2}\left(\hat{\phi}_{<}, \hat{\phi}_{>}\right)+S_{2}\left(\hat{\phi}_{m}\right) \text {. }
$$

Now given that $\mathcal{P}_{0}\left\{\hat{\phi}_{m}\right\}=Z_{0, m}^{-1} \exp \left\{-S_{2}\left(\hat{\phi}_{m}\right)\right\}$ is the probability distribution generated by $S_{2}\left(\hat{\phi}_{m}\right)$, let $\langle\ldots\rangle_{0}$ denote averages taken with respect to this distribution. Using this notation, Eq. (B1) becomes

$$
\begin{aligned}
& \int \mathcal{D} \hat{\phi}_{m} \exp \left\{-S\left(\hat{\phi}_{<}, \hat{\phi}_{>}, \hat{\phi}_{m}\right)\right\} \\
& =\exp \left\{-S_{2}\left(\hat{\phi}_{<}, \hat{\phi}_{>}\right)\right\}\left\langle\exp \left\{-S_{r}\right\}\right\rangle_{0} .
\end{aligned}
$$

Note that we have absorbed any contribution from $Z_{0, m}$ into the path-integral measure.

Equation (B3) may be (approximately) calculated by expansion techniques. Two techniques that are frequently used are the cumulant expansion $[9,10]$ and Feynman diagram expansions [7]. Although Feynman diagram expansion methods permit infinite resummations more easily than the cumulant expansion, we make use of the cumulant expansion. However, we will still illustrate the nonvanishing Feynman dia- grams that contribute to the cumulant expansion. Upon applying the cumulant expansion to Eq. (B3), we find that

$$
\left\langle\exp \left\{-S_{r}\right\}\right\rangle_{0}=\exp \left\{-\left\langle S_{r}\right\rangle_{0}+\frac{1}{2}\left[\left\langle S_{r}^{2}\right\rangle_{0}-\left\langle S_{r}\right\rangle_{0}^{2}\right]-\ldots\right\} \text {. }
$$

The first term in the exponent on the right-hand side of Eq. (B4) is the first cumulant while the second term (in square brackets) is the second cumulant. If we take $S^{\prime}$ to be the exponent on the right-hand side of Eq. (B4) then the Feynman diagram representation of $S^{\prime}$ is given by

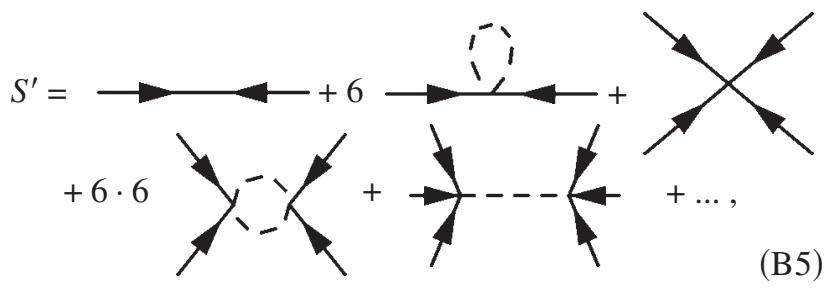

where the solid propagators represent the $\hat{\phi}_{<}$and $\hat{\phi}_{>}$propagators, while the dashed propagator represents the $\hat{\phi}_{m}$ propagator. We will use the first and second cumulants (and diagrammatics) to derive the RG equations in the following sections.

\section{Two-point couplings}

If we consider having a mass term (i.e., $\nabla^{2} \rightarrow \nabla^{2}-m^{2}$ ), then after integrating out $\hat{\phi}_{m}$ and rescaling $\hat{\phi}_{>}$and $\hat{\phi}_{<}$according Eq. (28), the bare two-point propagator (the Green's function) becomes

$$
\begin{aligned}
\longrightarrow & =\hat{\phi}_{<} \longrightarrow \hat{\phi}_{<}+\hat{\phi}_{>} \longrightarrow \longrightarrow \hat{\phi}_{>} \\
& =\frac{1}{2}\left(\int_{q} q^{2}|\hat{\varphi}(q)|^{2}+\Lambda^{-2} \int_{|q|<1} m_{<}^{2}|\hat{\varphi}(q)|^{2}+\Lambda^{2} \int_{|q|>1} m_{>}^{2}|\hat{\varphi}(q)|^{2}\right) .
\end{aligned}
$$

To simplify the notation, we denote the free propagator by $G_{0}(p)$. Similarly, for $|p|<1$ we denote the propagator by $G_{0}^{<}(p)$ and we do similarly for $|p|>1$. At one loop, we obtain

$$
\begin{aligned}
\longrightarrow & =\frac{1}{4 !}\left(\Lambda^{-2}\left[\lambda_{(4,0)} \int_{\Lambda<|p|<1} G_{0}^{<}(p)+\lambda_{(2,2)} \int_{1<|p|<\lambda^{-1}} G_{0}^{>}(p)\right] \int_{|q|<1}|\hat{\varphi}(q)|^{2}\right. \\
& \left.+\Lambda^{2}\left[\lambda_{(2,2)} \int_{\Lambda<|p|<1} G_{0}^{<}(p)+\lambda_{(0,4)} \int_{1<|p|<\lambda^{-1}} G_{0}^{>}(p)\right] \int_{|q|>1}|\hat{\varphi}(q)|^{2}\right) \\
= & \frac{\lambda S_{D}}{4 !(2 \pi)^{D}}(1-\Lambda)\left(\left[\frac{\lambda_{(4,0)}}{m_{<}^{2}+1}+\frac{\lambda_{(2,2)}}{m_{>}^{2}+1}\right] \int_{|q|<1}|\hat{\varphi}(q)|^{2}+\left[\frac{\lambda_{(2,2)}}{m_{<}^{2}+1}+\frac{\lambda_{(0,4)}}{m_{>}^{2}+1}\right] \int_{|q|>1}|\hat{\varphi}(q)|^{2}\right),
\end{aligned}
$$

where $G_{0}(p)$ is the free propagator and $S_{D}$ is the area of the $D$-dimensional unit sphere.

By combining Eqs. (B4)-(B7) and using $\Lambda=e^{-d l}$, we arrive at the RG equations [Eqs. (35) and (36)]

$$
\partial_{l} m_{<}^{2}=2 m_{<}^{2}+\frac{S_{D}}{2(2 \pi)^{D}}\left(\frac{\lambda_{(4,0)}}{m_{<}^{2}+1}+\frac{\lambda_{(2,2)}}{m_{>}^{2}+1}\right)+\mathcal{O}\left(\lambda^{2}\right),
$$




$$
\partial_{l} m_{>}^{2}=-2 m_{>}^{2}+\frac{S_{D}}{2(2 \pi)^{D}}\left(\frac{\lambda_{(2,2)}}{m_{<}^{2}+1}+\frac{\lambda_{(0,4)}}{m_{>}^{2}+1}\right)+\mathcal{O}\left(\lambda^{2}\right) .
$$

\section{Four-point couplings}

The decoupling of the wave-vector regimes, while explicit in the calculation of the two-point couplings, is more subtle in the calculation of the four-point couplings. However, it is still manifestly evident at tree level. Consequently, as in the previous section, we will first calculate the rescalings for the bare four-point vertex.

$$
\chi_{\phi_{<}}^{\hat{\phi}_{<}}=\Lambda^{D-4} \frac{\lambda_{(4,0)}}{4 !(2 \pi)^{4 D}} \int_{\left|p_{i}\right|<1} \prod_{i} \frac{d^{D} p_{i}}{(2 \pi)^{D}}(2 \pi)^{D} \delta^{D}\left(\sum_{j=1}^{4} p_{j}\right) \varphi\left(p_{1}\right) \varphi\left(p_{2}\right) \varphi\left(p_{3}\right) \varphi\left(p_{4}\right),
$$

$$
\prod_{\hat{\phi}_{<}}=\Lambda^{2(D-1)} \frac{\lambda_{(3,1)}}{4 !(2 \pi)^{4 D}} \int_{\left|p_{i}\right|<1} \prod_{i=1}^{3} \frac{d^{D} p_{i}}{(2 \pi)^{D}} \int_{\left|p_{4}\right|>1} \frac{d^{D} p_{4}}{(2 \pi)^{D}}(2 \pi)^{D} \delta^{D}\left(\sum_{j=1}^{4} p_{j}\right) \varphi\left(p_{1}\right) \varphi\left(p_{2}\right) \varphi\left(p_{3}\right) \varphi\left(p_{4}\right),
$$

$$
\prod_{\phi_{<}}^{\phi_{\phi_{<}}}=\Lambda^{D} \frac{\lambda_{(2,2)}}{4 !(2 \pi)^{4 D}} \int_{\left|p_{i}\right|<1} \prod_{i=1}^{2} \frac{d^{D} p_{i}}{(2 \pi)^{D}} \int_{\left|p_{k}\right|>1} \prod_{k=3}^{4} \frac{d^{D} p_{k}}{(2 \pi)^{D}}(2 \pi)^{D} \delta^{D}\left(\sum_{j=1}^{4} p_{j}\right) \varphi\left(p_{1}\right) \varphi\left(p_{2}\right) \varphi\left(p_{3}\right) \varphi\left(p_{4}\right),
$$

$$
\overbrace{\hat{\phi}>}^{\hat{\phi}_{>}}=\Lambda^{2} \frac{\lambda_{(1,3)}}{4 !(2 \pi)^{4 D}} \int_{\left|p_{i}\right|>1} \prod_{i=2}^{4} \frac{d^{D} p_{i}}{(2 \pi)^{D}} \int_{\left|p_{1}\right|<1} \frac{d^{D} p_{1}}{(2 \pi)^{D}}(2 \pi)^{D} \delta^{D}\left(\sum_{j=1}^{4} p_{j}\right) \varphi\left(p_{1}\right) \varphi\left(p_{2}\right) \varphi\left(p_{3}\right) \varphi\left(p_{4}\right),
$$

$$
\chi_{\phi_{>}}^{\hat{\phi}_{>}}=\Lambda^{4-D} \frac{\lambda_{(0,4)}}{4 !(2 \pi)^{4 D}} \int_{\left|p_{i}\right|>1} \prod_{i} \frac{d^{D} p_{i}}{(2 \pi)^{D}}(2 \pi)^{D} \delta^{D}\left(\sum_{j=1}^{4} p_{j}\right) \varphi\left(p_{1}\right) \varphi\left(p_{2}\right) \varphi\left(p_{3}\right) \varphi\left(p_{4}\right) .
$$

At one loop, a sample calculation with only $\hat{\phi}_{<}$on the external legs of the four-point diagram produces

$$
\begin{aligned}
{ }_{\phi} & \\
= & \left(\frac{1}{4 !}\right)^{2}(2 \pi)^{-4 D} \int_{\left|q_{i}\right|<\Lambda} \int_{\Lambda<\left|Q_{i}\right|<\Lambda^{-1}} \lambda\left(q_{1}, q_{2}, Q_{1}, Q_{2}\right)(2 \pi)^{D} \delta^{D}\left(q_{1}+q_{2}+Q_{1}+Q_{2}\right) \\
& \times \lambda\left(q_{3}, q_{4}, Q_{3}, Q_{4}\right)(2 \pi)^{D} \delta^{D}\left(q_{3}+q_{4}+Q_{3}+Q_{4}\right)(2 \pi)^{D} \delta^{D}\left(Q_{1}+Q_{3}\right) G_{0}\left(Q_{1}\right) \\
& \times(2 \pi)^{D} \delta^{D}\left(Q_{2}+Q_{4}\right) G_{0}\left(Q_{2}\right) \varphi\left(q_{1}\right) \varphi\left(q_{2}\right) \varphi\left(q_{3}\right) \varphi\left(q_{4}\right),
\end{aligned}
$$

$$
\begin{aligned}
\approx & \left(\frac{1}{4 !(2 \pi)^{D / 2}}\right)^{2} \int_{\left|q_{i}\right|<1}\left[\int_{\Lambda \leq\left|Q_{1}\right|,\left|Q_{2}\right|<1} \lambda_{(4,0)}^{2} G_{0}^{<}\left(Q_{1}\right) G_{0}^{<}\left(Q_{2}\right)\right. \\
& \left.+2 \int_{\Lambda \leq\left|Q_{1}\right|<1} \int_{1 \leq\left|Q_{2}\right| \leq \Lambda^{-1}} \lambda_{(3,1)}^{2} G_{0}^{<}\left(Q_{1}\right) G_{0}^{>}\left(Q_{2}\right)+\int_{1 \leq\left|Q_{1}\right|,\left|Q_{2}\right| \leq 1} \lambda_{(2,2)}^{2} G_{0}^{>}\left(Q_{1}\right) G_{0}^{>}\left(Q_{2}\right)\right] \\
& \times \delta^{D}\left(q_{1}+q_{2}+Q_{1}+Q_{2}\right)(2 \pi)^{D} \delta^{D}\left(q_{3}+q_{4}-Q_{1}-Q_{2}\right) \varphi\left(q_{1}\right) \varphi\left(q_{2}\right) \varphi\left(q_{3}\right) \varphi\left(q_{4}\right) .
\end{aligned}
$$

From Eq. (B17), there is an acquired wave-vector dependence. For the sake of comparison with equations, we only wish to derive the (naive) flow equations for the constant part of $\lambda\left(q_{1}, q_{2}, q_{3}, q_{4}\right)$. Thus, we obtain 


$$
\begin{aligned}
& \left.+\lambda_{(2,2)}^{2}\left(1+m_{>}^{2}\right)^{-2}\right] \int_{\left|q_{i}\right|<1}(2 \pi)^{D} \delta^{D}\left(\sum_{j=1}^{4} q_{j}\right) \varphi\left(q_{1}\right) \varphi\left(q_{2}\right) \varphi\left(q_{3}\right) \varphi\left(q_{4}\right) .
\end{aligned}
$$

Combining Eqs. (B4), (B5), (B11)-(B15), and (B18) and using $\Lambda=e^{-d l}$ yields the RG equations given in Eq. (30).

[1] L. F. Cugliandolo, J. Kurchan, and L. Peliti, Phys. Rev. E 55, 3898 (1997).

[2] P. C. Martin, E. D. Siggia, and H. A. Rose, Phys. Rev. A 8, 423 (1973).

[3] F. Cooper, A. Khare, and H. Rose, Phys. Lett. B 515, 463 (2001).

[4] J. Zanella and E. Calzetta, Phys. Rev. E 66, 036134 (2002).

[5] K. Chou, Z. Su, B. Hao, and L. Yu, Phys. Rep. 118, 1 (1985).

[6] J. Zinn-Justin, Quantum Field Theory and Critical Phenomena, 3rd ed. (Oxford University Press, New York, 1996).

[7] K. G. Wilson and J. Kogut, Phys. Rep. 12, 75 (1974).

[8] K. G. Wilson, Rev. Mod. Phys. 47, 773 (1975).

[9] N. Goldenfeld, Lectures on Phase Transitions and the Renormalization Group (Perseus, Reading, MA, 1992).

[10] R. Shankar, Rev. Mod. Phys. 66, 129 (1994).

[11] A. Degenhard and J. Rodríguez-Laguna, J. Stat. Phys. 106, 1093 (2002).

[12] J. Elf, J. Paulsson, O. G. Berg, and M. Ehrenberg, Biophys. J. 84, 154 (2003).

[13] J. Paulsson, Nature (London) 427, 415 (2004).

[14] R. P. Feynman and F. L. Vernon, Ann. Phys. (N.Y.) 24, 118 (1963).

[15] A. O. Caldeira and A. J. Leggett, Physica A 121, 587 (1983).

[16] L.-Y. Chen, N. Goldenfeld, and Y. Oono, Phys. Rev. E 54, 376 (1996).

[17] A. Degenhard and J. Rodríguez-Laguna, Multiscale Model. Simul. 4, 641 (2005).

[18] K. Glover, Int. J. Control 39, 1115 (1984).
[19] V. V. Peller, Hankel Operators and Their Applications (Springer, New York, 2003).

[20] G. E. Dullerud and F. Paganini, A Course in Robust Control Theory (Springer, New York, 2000).

[21] J. M. A. Scherpen, Syst. Control Lett. 21, 143 (1993).

[22] J. Müller and J. Rau, Phys. Lett. B 386, 274 (1996).

[23] V. Bach, J. Fröhlich, and I. M. Sigal, Adv. Math. 137, 205 (1998).

[24] V. Bach, T. Chen, J. Frölich, and I. M. Sigal, J. Funct. Anal. 203, 44 (2003).

[25] G. A. Baker, Jr., and P. Graves-Morris, Padé Approximants, 2nd ed. (Cambridge University Press, New York, 1996).

[26] G. Battle, Commun. Math. Phys. 110, 601 (1987).

[27] G. Battle, Commun. Math. Phys. 114, 93 (1988).

[28] S. R. White, Phys. Rev. Lett. 69, 2863 (1992).

[29] S. R. White, Phys. Rev. B 48, 10345 (1993).

[30] Whenever the poles of the resolvent are on the imaginary axis, a pole prescription must be supplied. We deform the poles of the resolvent into the left-hand plane.

[31] $\dot{\xi}$ is pathologically fast compared to $\xi$.

[32] K. I. Matsuba and K. Nozaki, Phys. Rev. E 56, R4926 (1997).

[33] J. Bricmont, A. Kupiainen, and A. Schenkel, Commun. Math. Phys. 221, 101 (2001).

[34] R. Boesch, P. Stancioff, and C. R. Willis, Phys. Rev. B 38, 6713 (1988).

[35] T. A. Brun and J. B. Hartle, Phys. Rev. D 60, 123503 (1999).

[36] N. Maïzi, Analysis and Optimization of Systems: State and Frequency Domain Approaches for Infinite-Dimensional Systems (Springer-Verlag, Berlin, 1993), pp. 585-597. 